\title{
Virus infection speeds: Theory versus experiment
}

\author{
Daniel R. Amor and Joaquim Fort \\ Complex Systems Laboratory, Departament de Física, Universitat de Girona, 17071 Girona, Catalonia, Spain \\ (Received 31 July 2010; revised manuscript received 15 November 2010; published 14 December 2010)
}

\begin{abstract}
In order to explain the speed of Vesicular Stomatitis Virus (VSV) infections, we develop a simple model that improves previous approaches to the propagation of virus infections. For VSV infections, we find that the delay time elapsed between the adsorption of a viral particle into a cell and the release of its progeny has a very important effect. Moreover, this delay time makes the adsorption rate essentially irrelevant in order to predict VSV infection speeds. Numerical simulations are in agreement with the analytical results. Our model satisfactorily explains the experimentally measured speeds of VSV infections.
\end{abstract}

DOI: 10.1103/PhysRevE.82.061905

PACS number(s): 87.23.Cc, 89.20.-a, 89.75.Fb

\section{INTRODUCTION}

Mathematical models of infectious diseases are a field that has advanced substantially during the last decades [1]. It makes use of many methods from Physics, e.g., reactiondiffusion equations and front speed computations. On the other hand, in the last century vaccination has become one of the best tools against infection spreading, and some models including vaccination strategies have been developed recently $[2,3]$. Moreover, new computer technology advances have played an important role on infection spreading research, since they have made it possible to introduce novel methods such as complex networks [4-6]. However, in addition with macroscopic epidemiology studies $[1-3,5]$, the effects of infectious diseases at a cellular levels (as well as microscopic viral infection front propagation) are also of scientific interest [7-12].

When a virus infects a cell, some time $\tau$ elapses before the new generation of viruses is released from the cell. Some years ago, it was shown that this delay time $\tau$ has an important effect and can satisfactory explain the infection front speeds of T7 viruses infecting E. Coli bacteria [13-15]. In Ref. [15], we mentioned that it should be possible to apply the same model to Vesicular Stomatitis Viruses (VSV), which replicate on mammalian or insect cells (not on bacteria), because there is again a delay due to the time elapsed between virus adsorption into a cell and the release of its progeny. Detailed measurements of infection front speeds for VSV were not available when we developed our models $[8,13]$, but such measurements have been performed recently $[16,17]$.

Some very recent models for the spread of VSV infections have been proposed by Haseltine et al. [17]. Their models include the reaction-diffusion process, as well as some additional relevant biological phenomena, in order to explain the experimental images of this phenomenon. In this paper we revisit the approach by Haseltine et al. [17] to reduce the complexity of the models, and to better account for the experimental speeds. We will present a simple model, using as few unknown parameters as possible, and compare its predicted front speeds to VSV experimental data [16]. We will show that it is necessary to take it into account the effect of the delay time $\tau$ in order to explain properly the front speeds of VSV infections.
The plan of this paper is as follows. In Sec. II we present a time-delayed model using as few free or adjustable parameters as possible. In Sec. III we derive some approximate explicit equations for the front speed. In Sec. IV, we perform numerical simulations to check the validity of our theoretical model. In Sec. V we compare to experimental data. Section VI is devoted to concluding remarks, especially the importance of the delay time $\tau$ for VSV infections. We will also find that this delay-time effect leads to the front speed being approximately independent of the adsorption constant $k_{1}$ over four orders of magnitude. This is very important in order to compare predicted speeds to experimental ones because the value of $k_{1}$ is very uncertain.

\section{MODEL}

The infection and virus replication processes can be summarized by the reactions

$$
\begin{aligned}
& k_{1} \quad k_{2} \\
& V+C \rightarrow I \rightarrow Y V .
\end{aligned}
$$

In Eq. (1), $k_{1}$ is the adsorption rate of viruses $V$ into uninfected cells $C, k_{2}$ is the rate constant for the death of infected cells $I$, and the yield $Y$ is the number of new viruses produced per infected cell. In contrast to some previous papers $[8,13-15]$, here we use the symbol $C$ instead of $B$ because VSV infections propagate on mammalian or insect cells (not on bacteria, in contrast to T7 infections).

The experiments reported in Refs. [16,17] were performed in agar, such that cells are immobilized and only viruses diffuse. The extracellular model by Haseltine et al. [see Eq. (5a) in Ref. [17]] uses the following reactiondiffusion equations for the concentrations at large infected distances, $r \rightarrow \infty$ (where $r=0$ corresponds to the initial inoculation point of viruses into a medium of uninfected cells) $[18,19]$,

$$
\begin{aligned}
\frac{\partial[V](r, t)}{\partial t}= & D \frac{\partial^{2}[V](r, t)}{\partial r^{2}}-k_{1}[V][C](r, t)+k_{2} Y[I](r, t), \\
& \frac{\partial[C](r, t)}{\partial t}=-k_{1}[C](r, t)[V](r, t),
\end{aligned}
$$




$$
\frac{\partial[I](r, t)}{\partial t}=k_{1}[V](r, t)[C](r, t)-k_{2}[I](r, t),
$$

where $D$ is the diffusion coefficient, $[\ldots]$ denotes concentration and $t$ is the time. The set of Eqs. (2)-(4) can be further improved for two reasons, which we now discuss.

First, Eq. (4) implies a virus dynamics that does not agree with experimental data in homogeneous media [see Eq. (A4) and the text below it in Appendix A]. In order to improve this point, it has been proposed [13] that $k_{2}[I](r, t)$ in Eqs. (2) and (4) should be replaced by a logistic-type growth function, namely, $k_{2}[I](r, t)\left\{1-[I](r, t) / I_{M A X}\right\}$, which leads to a virus dynamics that agrees very well with one-step growth experimental data (see Appendix A). Such an improved model is therefore described, instead of by Eqs. (2)-(4), by the following set:

$$
\begin{aligned}
& \frac{\partial[V](r, t)}{\partial t}= D \frac{\partial^{2}[V](r, t)}{\partial r^{2}}-k_{1}[V][C](r, t)+k_{2} Y[I](r, t) \\
& \times\left(1-\frac{[I](r, t)}{I_{M A X}}\right), \\
& \frac{\partial[C](r, t)}{\partial t}=-k_{1}[C](r, t)[V](r, t), \\
& \frac{\partial[I](r, t)}{\partial t}=k_{1}[V](r, t)[C](r, t)-k_{2}[I](r, t)\left(1-\frac{[I](r, t)}{I_{M A X}}\right) .
\end{aligned}
$$

This set is more reasonable than Eqs. (2)-(4) because it agrees better with experimental data in homogeneous media (see Appendix A), but we would like to stress that this point does not affect the analytical results in the present paper because the infection speeds that we will derive would be the same if the last term in Eqs. (5) and (7) were neglected (see Appendix A).

A second, much more important improvement can be made. The diffusion dynamics in both Eqs. (5) and (2) (left side and first term in the right side) is Fickian or nondelayed. This means that it does not take into account the effect of the time interval $\tau$ during which a virus does not move appreciably because it is inside a cell. The parameter $\tau$ is the time interval elapsed from the adsorption of a virus into a cell until the virus has reproduced inside it and the virus progeny have left the cell. Obviously, this effect will slow down the propagation of virus fronts. Indeed, it has been previously shown that taking into account this delay time is essential to find good agreement with the experimental observations in other kinds of virus infections [13]. For this reason, Eq. (5) must be replaced by a time-delayed diffusion equation [20], namely, (see Appendix B for a derivation)

$$
\begin{aligned}
& \frac{\partial[V](r, t)}{\partial t}+\frac{\tau}{2} \frac{\partial^{2}[V](r, t)}{\partial t^{2}} \\
& \quad=D \frac{\partial^{2}[V](r, t)}{\partial r^{2}}+F(r, t)+\left.\frac{\tau}{2} \frac{\partial F(r, t)}{\partial t}\right|_{g},
\end{aligned}
$$

where the virus growth function $F(r, t)$ accounts for all growth processes affecting the virus population density $[\mathrm{V}]$, i.e.,

$$
\begin{aligned}
F(r, t) \equiv & \left.\frac{\partial[V](r, t)}{\partial t}\right|_{g} \\
= & -k_{1}[V](r, t)[C](r, t)+k_{2} Y[I](r, t) \\
& \times\left(1-\frac{[I](r, t)}{I_{M A X}}\right) .
\end{aligned}
$$

Note that Eq. (8) simplifies to Eq. (5) if the effect of the delay time is neglected $(\tau=0)$.

The symbol $\left.\ldots\right|_{g}$ indicates that the corresponding time derivatives in Eqs. (8) and (9) take into account exclusively growth (i.e., reactive) but not diffusive processes. In a recent paper [21] it has been shown that taking this subindex properly into account improves previous results [22] for the speed of fronts (albeit Refs. [21,22] consider a simpler system with a single species, whereas here we have three species). For this very same reason, Eq. (8) here improves Eq. (6) in Ref. [13], and yields a different infection speed that will be derived below. In order to do so, let us further develop the last term in Eq. (8),

$$
\begin{aligned}
\left.\frac{\tau}{2} \cdot \frac{\partial F(r, t)}{\partial t}\right|_{g}= & -\left.\frac{\tau}{2} k_{1} \frac{\partial\{[V](r, t)[C](r, t)\}}{\partial t}\right|_{g}+\frac{\tau}{2} k_{2} Y \frac{\partial}{\partial t} \\
& \times\left.\left[[I](r, t)\left(1-\frac{[I](r, t)}{I_{M A X}}\right)\right]\right|_{g}
\end{aligned}
$$

From Eqs. (6) and (7), we can see that there are no diffusive processes affecting the time derivatives of neither uninfected $C$ nor infected cells $I$ (physically, this is due to the fact that cells are immobilized in agar in these experiments). Thus, in fact the symbol ... $\left.\right|_{g}$ is not required for the last term in Eq. (10), and the first term on the right can be written as $-\frac{\tau}{2} k_{1}[V]$ $\partial[C] / \partial t-\frac{\tau}{2} k_{1}[C] \partial[V] /\left.\partial t\right|_{g}$. Making use of Eq. (9), this allows us to rewrite Eq. (8) as

$$
\begin{aligned}
\frac{\partial[V](r, t)}{\partial t} & +\frac{\tau}{2} \frac{\partial^{2}[V](r, t)}{\partial t^{2}} \\
= & D \frac{\partial^{2}[V](r, t)}{\partial r^{2}}+F(r, t)-\frac{\tau}{2} k_{1}[V](r, t) \frac{\partial[C](r, t)}{\partial t} \\
& \quad-\frac{\tau}{2} k_{1}[C](r, t) F(r, t)+\frac{\tau}{2} k_{2} Y \frac{\partial}{\partial t}\left[[I](r, t)\left(1-\frac{[I](r, t)}{I_{M A X}}\right)\right] .
\end{aligned}
$$

This equation has three dependent variables, namely, $[V](r, t),[C](r, t)$, and $[I](r, t)$, so in order to analyze its solutions we need two additional equations, namely, Eqs. (6) and (7),

$$
\frac{\partial[C](r, t)}{\partial t}=-k_{1}[C](r, t)[V](r, t),
$$




$$
\frac{\partial[I](r, t)}{\partial t}=k_{1}[V](r, t)[C](r, t)-k_{2}[I](r, t)\left(1-\frac{[I](r, t)}{I_{M A X}}\right) .
$$

Our model will be based on the set of Eqs. (11)-(13). Before going ahead, however, we can see better the importance of the symbol $\left.\ldots\right|_{g}$ by noting that, if it had not been included in Eq. (8), Eq. (10) would be the same but without the symbols $\left.\ldots\right|_{g}$, so its first term on the right would be $-\frac{\tau}{2} k_{1}([V] \partial[C] / \partial t+[C] \partial[V] / \partial t)$ and, since the symbol $\left.\ldots\right|_{g}$ does not appear, we would be unable to substitute Eq. (9). Therefore, instead of Eq. (11) we would have obtained

$$
\begin{aligned}
\frac{\partial[V](r, t)}{\partial t} & +\frac{\tau}{2} \frac{\partial^{2}[V](r, t)}{\partial t^{2}} \\
= & D \frac{\partial^{2}[V](r, t)}{\partial r^{2}}+F(r, t)-\frac{\tau}{2} k_{1}[V](r, t) \frac{\partial[C](r, t)}{\partial t} \\
& \quad-\frac{\tau}{2} k_{1}[C] \frac{\partial[V](r, t)}{\partial t}+\frac{\tau}{2} k_{2} Y \frac{\partial}{\partial t}\left[[I](r, t)\left(1-\frac{[I](r, t)}{I_{M A X}}\right)\right] .
\end{aligned}
$$

This corresponds to the model in Refs. [13,14]. The fourth term in the right of Eq. (14) is improved by that in Eq. (11). The difference is that this term in Eq. (11) corresponds to the variation of $[V](r, t)$ due to adsorption and replication, whereas in Eq. (14) it also includes its variation due to diffusion [i.e., Eq. (14) uses $\frac{\partial[V](r, t)}{\partial t}$ instead of $\left.\left.\frac{\partial[V](r, t)}{\partial t}\right|_{g}=F(r, t)\right]$. For details, see the derivation of Eq. (8) in Appendix B in the present paper.

Our model will be based on the set of Eqs. (11)-(13). Their front speed will be derived below, and they will be integrated numerically in Sec. IV.

Equations (11)-(13) can be written in terms of dimensionless variables $\bar{C} \equiv[C] / C_{0}, \bar{V} \equiv[V] / C_{0}, \bar{I} \equiv[I] / C_{0}, \bar{t} \equiv k_{2} t$, and $\bar{r} \equiv r \sqrt{k_{2} / D}$ and dimensionless parameters $\bar{\tau} \equiv k_{2} \tau, \kappa$ $\equiv k_{1} C_{0} / k_{2}$, and $\overline{I_{M A X}}=I_{M A X} / C_{0}$, where $C_{0}$ is the initial cell concentration. Then Eqs. (11)-(13) become

$$
\frac{\bar{\tau}}{2} \bar{V}_{\overline{t t}}+\bar{V}_{\bar{t}}=\bar{V}_{\overline{r r}}+\bar{F}-\frac{\bar{\tau}}{2} \kappa \bar{V} \bar{C}_{\bar{t}}-\frac{\bar{\tau}}{2} \kappa \bar{F} \bar{C}+\frac{\bar{\tau}}{2} Y\left[\bar{I}\left(1-\frac{\bar{I}}{\bar{I}_{M A X}}\right)\right]_{\bar{t}},
$$

$$
\begin{gathered}
\bar{C}_{\bar{t}}=-\kappa \bar{V} \bar{C}, \\
\bar{I}_{\bar{t}}=\kappa \bar{V} \bar{C}-\bar{I}\left(1-\frac{\bar{I}}{\overline{I_{M A X}}}\right),
\end{gathered}
$$

where $\bar{F}$ is the dimensionless growth function defined as

$$
\bar{F}=-\kappa \bar{V} \bar{C}+Y \bar{I}\left(1-\frac{\bar{I}}{\overline{I_{M A X}}}\right) .
$$

For simplicity, in Eqs. (15)-(18) we have used de notation $\bar{V}_{\bar{t}}, \bar{C}_{\bar{t}}$, and $\bar{I}_{\bar{t}}$ to indicate, respectively, the partial time derivatives of $\bar{V}, \bar{C}$, and $\bar{I}$. Moreover, we have omitted the dependences of the dimensionless population densities and growth function [i.e., $\bar{V}(\bar{r}, \bar{t}), \bar{C}(\bar{r}, \bar{t}), \bar{I}(\bar{r}, \bar{t})$, and $\bar{F}(\bar{r}, \bar{t})$ appear as $\bar{V}$, $\bar{C}, \bar{I}$, and $\bar{F}$. In Eq. (15) $\bar{V}_{\overline{t t}}$ and $\bar{V}_{\overline{r r}}$ stand for the second partial time and second partial space derivatives of $\bar{V}$, respectively.

We look for solutions depending only on the new variable $\bar{z} \equiv \bar{r}-\bar{c} \bar{t}$, where $\bar{c}>0$ is the dimensionless wave front speed, which is related to dimensional speed $c$ by $\bar{c}=c / \sqrt{k_{2} D \text {. As }}$ usual, we linearize our Eqs. (15)-(18) around the unstable steady state $([V],[C],[I])=\left(0, C_{0}, 0\right)$, i.e., $(\bar{V}, \bar{C}, \bar{I})=\left(\epsilon_{V}, 1\right.$ $\left.-\epsilon_{C}, \epsilon_{I}\right)$, where $\vec{\epsilon}=\left(\epsilon_{V}, \epsilon_{C}, \epsilon_{I}\right)=\vec{\epsilon}_{0} \exp (-\lambda \vec{z})$. For nontrivial solutions $\left(\epsilon_{V}, \epsilon_{C}, \epsilon_{I}\right) \neq(0,0,0)$ to exist, the determinant of the matrix corresponding to the linearized set of three evolution equations must vanish. Therefore, the following characteristic equation must be satisfied:

$$
\begin{aligned}
& \lambda^{3}+ \frac{-\bar{c}^{2}(1+\beta)+1}{\bar{c}\left(1-\beta \bar{c}^{2}\right)} \lambda^{2}+\frac{\kappa(\beta \kappa-1)+\beta \kappa Y-1}{1-\beta \bar{c}^{2}} \lambda \\
&+\frac{(1-\beta \kappa)(\kappa Y-\kappa)}{\bar{c}\left(1-\beta \bar{c}^{2}\right)}=0 .
\end{aligned}
$$

For simplicity, we have introduced the parameter $\beta=\bar{\tau} / 2$. According to marginal stability analysis [23], the wave front speed can be calculated numerically from

$$
\bar{c}=\min _{\lambda>0}[\bar{c}(\lambda)],
$$

where $\bar{c}(\lambda)$ is given implicitly by Eq. (19).

\section{APPROXIMATE EXPLICIT EQUATIONS FOR THE SPEED}

In this section we derive explicit expressions for the dimensionless front speed $\bar{c}$. Its exact value is given by the implicit Eq. (20). Since an exact explicit equation for the speed would be very complicated, some assumptions and approximations will be made.

In order to avoid nonpositive values for concentrations, we must impose that the three solutions for $\lambda$ in Eq. (19) are real, so it must be satisfied that

$$
-4 C_{1}^{3} C_{3}+C_{1}^{2} C_{2}^{2}+18 C_{1} C_{2} C_{3}-4 C_{2}^{3}-27 C_{3}^{2} \geq 0,
$$

where $C_{1}, C_{2}$, and $C_{3}$ are the coefficients of second, first, and zeroth powers of $\lambda$, respectively. We rewrite condition (21) in terms of $\nu=\bar{c}^{2}$ and then we get

$$
a_{3} \nu^{3}+a_{2} \nu^{2}+a_{1} \nu+a_{0} \geq 0
$$

where the coefficients $a_{i}$ are easily derived from condition (21). However, the exact expressions for $a_{i}$ are rather long. Fortunately, they can be simplified under some simple approximations that take into account typical values of the parameters in the present paper, namely, a high value of the yield (e.g., $\left.Y=2.77 \times 10^{5}\right)$ and a small value of $\kappa$ (e.g., $\kappa$ $\left.=2.15 \times 10^{-3}\right)$. In Sec. $\mathrm{V}$ we will give a detailed discussion of these and other parameter values. Then the coefficients $a_{i}$ can be written as 


$$
\begin{aligned}
a_{3} \simeq 4 \kappa^{3} Y^{3} \beta^{4}-12 \kappa^{2} Y^{2} \beta^{3}+\left(\chi^{2} \kappa^{2} Y^{2}-18 \chi \omega \kappa^{2} Y^{2}\right. & a_{2} \simeq-4 \kappa^{3} Y^{3} \beta^{3}, \\
\left.-27 \omega^{2} \kappa^{2} Y^{2}\right) \beta^{2}+\left(-2 \chi^{2} \kappa Y+18 \chi \omega \kappa Y\right) \beta-4 \chi^{3} \omega \kappa Y, & a_{1} \simeq \kappa^{2} Y^{2} \beta^{2}, \\
(23) & a_{0} \simeq 4 \omega \kappa Y,
\end{aligned}
$$

$$
\begin{aligned}
a_{2} \simeq & -4 \kappa^{3} Y^{3} \beta^{3}+\left(-2 \chi \kappa^{2} Y^{2}+18 \omega \kappa^{2} Y^{2}+12 \kappa^{2} Y^{2}\right) \beta^{2} \\
& +\left(18 \chi \omega \kappa^{2} Y^{2}+54 \omega^{2} \kappa^{2} Y^{2}\right) \beta+12 \chi^{2} \omega \kappa Y-18 \chi \omega \kappa Y,
\end{aligned}
$$

$$
\begin{gathered}
a_{1} \simeq \kappa^{2} Y^{2} \beta^{2}-18 \omega \kappa^{2} Y^{2} \beta-27 \omega^{2} \kappa^{2} Y^{2}, \\
a_{0} \simeq 4 \omega \kappa Y,
\end{gathered}
$$

where we have just kept the highest-order power of $\beta$ for every coefficient $a_{i}$. The critical condition to obtain the propagation speed is given by Eq. (22) when the equality holds. Then, substituting Eqs. (27)-(30) into Eq. (22), it is easy to see that the only real, positive solution if $\beta \gg 1$ is simply

$$
\bar{c} \simeq \frac{1}{\sqrt{\beta}} .
$$

where $\chi=1+\beta, \omega=\kappa \beta-1$, we have assumed the typical values mentioned above and made the approximation $(Y-1)$ $\simeq Y$. Even at this point, an exact solution for the front speed would be extremely cumbersome. Therefore, below we present two special cases where a manageable expression for the front speed can be found. First, we deal with the case $\beta \gg 1$. Then, Eqs. (23)-(26) can be simplified as

$$
a_{3} \simeq 4 \kappa^{3} Y^{3} \beta^{4},
$$

Note the interesting fact that the velocity is seen to be independent of $\kappa$ (hence, independent of $k_{1}$ ) and $Y$ in this limit. In Sec. V we will check this point by means of numerical simulations and discuss it in more detail.

On the other hand, we can easily obtain a solution for the nondelayed case $(\tau=0)$. Then $\beta=0$ and all of the terms containing $\beta$ in Eqs. (23)-(26) vanish. We substitute the resulting coefficients $a_{i}$ in Eq. (22) and obtain

$$
\begin{aligned}
\bar{c}_{\beta=0}= & \left(-\frac{\delta_{2}}{3 \delta_{3}}-\frac{2^{1 / 3}\left(-\delta_{2}^{2}+3 \delta_{3} \delta_{1} \kappa Y\right)}{3 \delta_{3}\left\{-2 \delta_{2}^{3}-\delta_{3}^{3} \delta_{1}+9 \delta_{3} \delta_{2} \delta_{1} \kappa Y+\left[\delta_{3}\left(-\delta_{2}^{2}+3 \delta_{3} \delta_{1} \kappa Y\right)^{3}+\left(-2 \delta_{2}^{3}-\delta_{3}^{3} \delta_{1}+9 \delta_{3} \delta_{2} \delta_{1} \kappa Y\right)^{2}\right]^{1 / 2}\right\}^{1 / 3}}\right. \\
& \left.+\frac{\left\{-2 \delta_{2}^{3}-\delta_{3}^{3} \delta_{1}+9 \delta_{3} \delta_{2} \delta_{1} \kappa Y+\left[\delta_{3}\left(-\delta_{2}^{2}+3 \delta_{3} \delta_{1} \kappa Y\right)^{3}+\left(-2 \delta_{2}^{3}-\delta_{3}^{3} \delta_{1}+9 \delta_{3} \delta_{2} \delta_{1} \kappa Y\right)^{2}\right]^{1 / 2}\right\}^{1 / 3}}{3 \cdot 2^{1 / 3} \delta_{3}}\right)^{1 / 2},
\end{aligned}
$$

where $\delta_{3}=4, \delta_{2}=6$, and $\delta_{1}=-27$.

\section{NUMERICAL SIMULATIONS}

We have integrated numerically the set of Eqs. (15)-(17), in order to check the exact speeds obtained from Eqs. (19) and (20) and the approximate speeds from Eqs. (31) and (32). Numerical simulations are also interesting because they make it possible to obtain not only the front speed, but also profiles for the concentrations of the three species, namely, $[\mathrm{V}]$, $[\mathrm{C}]$, and $[I]$.

In the experiments that we want to explain, the virus concentration of the initial inoculum is [17]

$$
[V]_{0}= \begin{cases}v_{0}, & r<0.075 \mathrm{~cm}, \\ \left(1-\frac{20}{\mathrm{~cm}}(r-0.075 \mathrm{~cm})\right) v_{0}, & 0.075 \mathrm{~cm} \leq r \leq 0.125 \mathrm{~cm}, \\ 0, & r>0.125 \mathrm{~cm},\end{cases}
$$

where $[\mathrm{V}]_{0}$ is the concentration of viruses at $t=0$ and $v_{0}$ $=9.3 \times 10^{8} / \mathrm{ml}$.

The initial condition (33), together with $[C]=C_{0}$ everywhere at $t=0$ and $[I]=0$ everywhere at $t=0$, was used in all simulations. We used finite differences to approximate the partial derivatives in Eqs. (15)-(17). Typical values for the space and time steps used in our simulations [e.g., in Fig. 1 and most of the simulations in Fig. 2(b)] were $1.5 \mathrm{~s}$ and $1 \times 10^{-5} \mathrm{~cm}$, respectively [dimensionless values for these steps were computed in order to use Eqs. (15)-(17)]. However, in some cases a higher resolution was required in order to find good agreement with the theoretical front speeds. When high values of the parameters $(Y, k$, or $\tau)$ were explored, the steps of space and time had to be reduced to $1 \times 10^{-6} \mathrm{~cm}$ and below $0.3 \mathrm{~s}$, respectively. Moreover, the same high resolution was required when very low values of the same parameters were used. Such step reductions lead to a substantial increase in computing time. Such higher reso- 


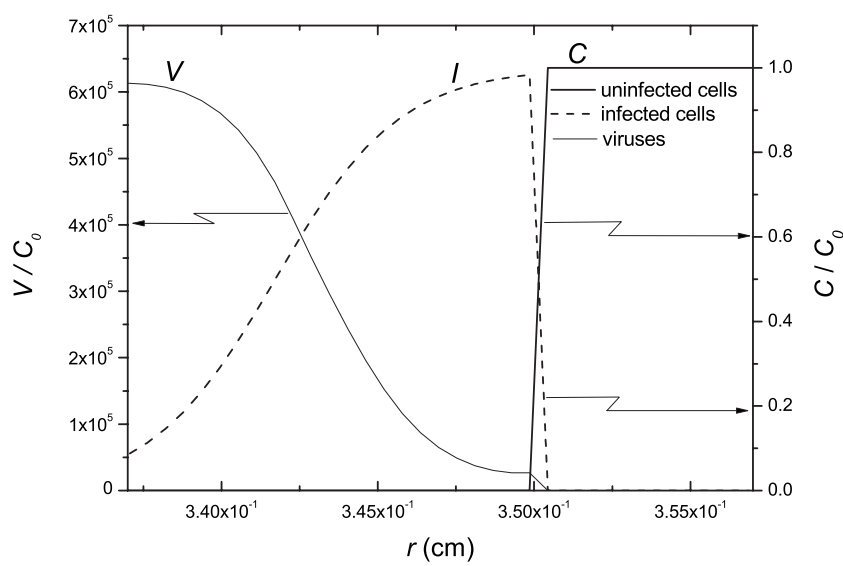

FIG. 1. Radial profiles of the concentrations $[V],[C]$, and $[I]$ after $48 \mathrm{~h}$ of infection, obtained from numerical simulations. The parameter values used are $C_{0}=3.8 \times 10^{7} / \mathrm{ml}, Y=2.77 \times 10^{5}$, $k_{1}=1.4 \times 10^{-10} \mathrm{~cm}^{3} / \mathrm{h}, \quad k_{2}=2.47 / \mathrm{h}, \quad \tau=8.1 \mathrm{~h} \quad$ and $\quad D=8.37$ $\times 10^{-5} \mathrm{~cm}^{2} / \mathrm{h}$ (see Sec. V for a discussion on these values).

lution was needed in some of the simulations in Figs. 2(a) and 3 .

Figure 1 depicts an example of the concentration profiles $[V],[C]$, and $[I]$ after $48 \mathrm{~h}$ of infection. In Fig. 1 we observe that the profile of the uninfected cells $C$ has the shape of a front (full curve), whereas that of the infected cells $I$ has the shape of a pulse. This was to be expected intuitively, because the infection [first reaction in Eq. (1)] decreases the number of uninfected cells $C$, which transform into infected cells $I$ and eventually die. On the other hand, their death releases new viruses $V$ [second reaction in Eq. (1)] after some delay time $\tau$, which explains the fact that the virus profile lags substantially behind that of infected cells $I$ (in contrast to what is observed in numerical simulations of nondelayed models [24]). Finally, let us compute the distance traveled by the infection front during $48 \mathrm{~h}$. This should be $48 \mathrm{~h}$ times $0.053 \mathrm{~cm} / \mathrm{h}$ [from Fig. 2(a), rhombus with $\tau \simeq 8$ h], i.e., 0.25 $\mathrm{cm}$. Adding $0.10 \mathrm{~cm}$ from the initial condition (33) we obtain $0.35 \mathrm{~cm}$ for the front position, in agreement with Fig. 1 .

\section{COMPARISON TO EXPERIMENT}

In this section we apply our model to the specific case of a VSV focal infection spreading in Baby Hamster Kidney (BHK) cells. Experimental results of this biophysical system, including the observed front speeds, have been published in Ref. [16]. Below we compare the observed speed range to both simulations and analytical solutions of our model.

First, we briefly discuss the parameter values used in our computations. Since we have not found any value for $I_{M A X}$ in the VSV experiments [16], we assume that $I_{M A X} \simeq C_{0}$ (in agreement with Fig. 1), i.e., that viruses are able to infect almost all of the cells at large enough times after the infection. The value of $I_{M A X}$ is thus $I_{M A X} \simeq C_{0}=3.8 \times 10^{7} / \mathrm{ml}$ [17].

The rate constant for the death of infected cells, $k_{2}$, and the virus yield, $Y$, can be easily derived from the one-step growth data of VSV on BHK cells (Fig. 3(a) in [17]) by fitting the logistic function [Eq. (A11) in Appendix A]. This
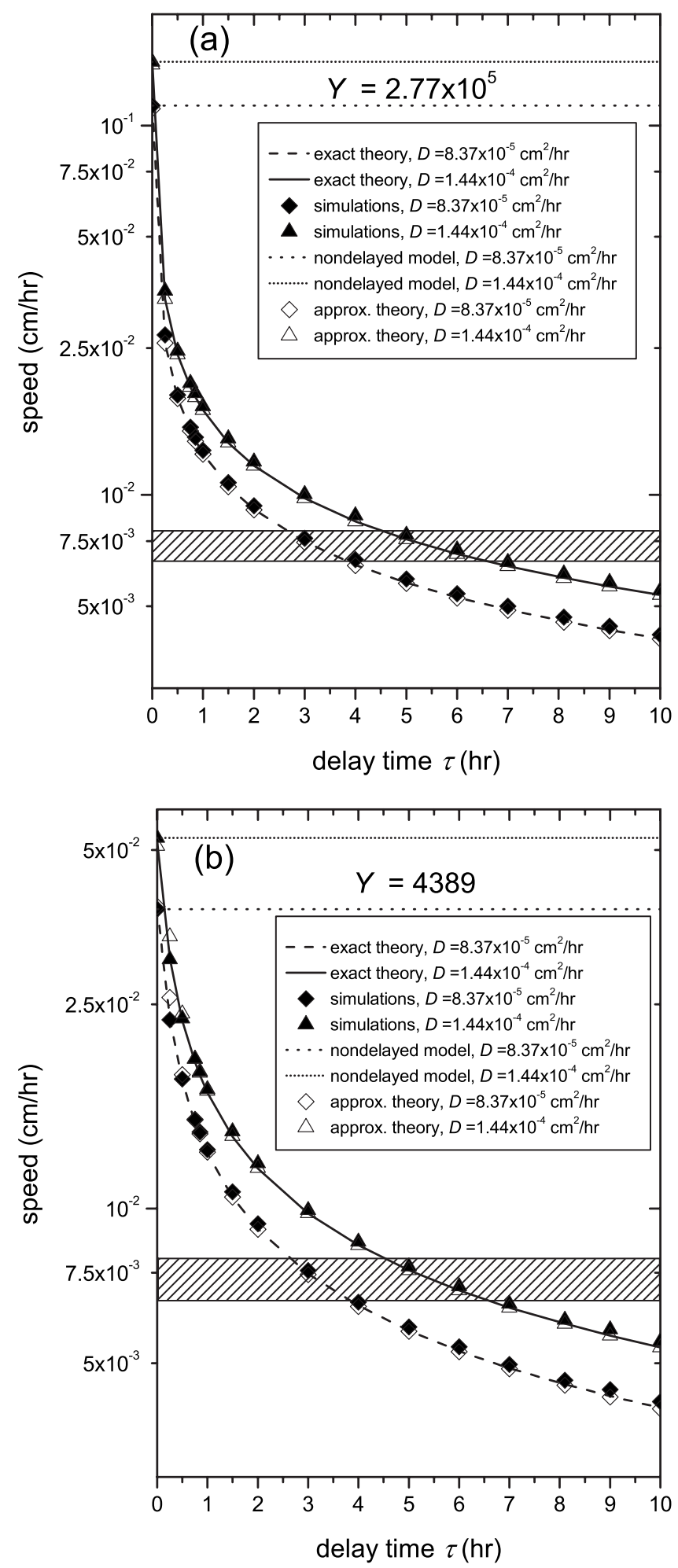

FIG. 2. Predicted front speed versus the delay time $\tau$, for (a) the value $Y=2.77 \times 10^{5}$ proposed in this paper and (b) the value $Y$ $=4389$ proposed in Ref. [17]. The dotted horizontal lines correspond to the nondelayed model $(\tau=0)$ and the hatched rectangle to the experimental range, namely, $(6.6-8.0) \times 10^{-3} \mathrm{~cm} / \mathrm{h}$ [27]. The curves are the analytical results from Eqs. (19) and (20) and the full symbols are the numerical simulations. The empty symbols indicate the approximate analytical speed results. The parameter values used are $C_{0}=3.8 \times 10^{7} / \mathrm{ml}, k_{1}=1.4 \times 10^{-10} \mathrm{~cm}^{3} / \mathrm{h}$, and $k_{2}=2.47 / \mathrm{h}$. 


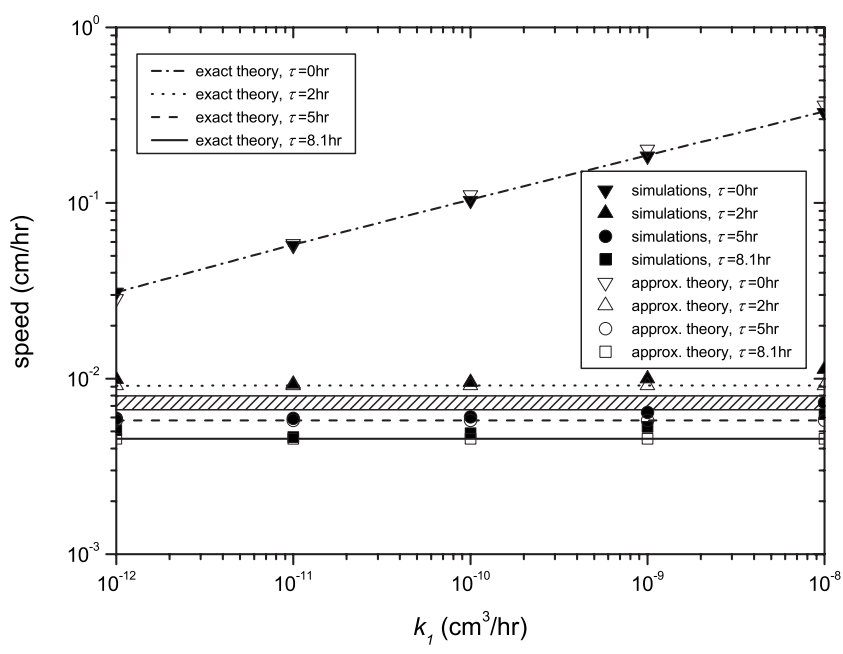

FIG. 3. Front speed versus adsorption rate $k_{1}$ for several values of the delay time. The hatched rectangle corresponds to the experimental range, namely, $(6.6-8.0) \times 10^{-3} \mathrm{~cm} / \mathrm{h}$ [27]. The curves correspond to the analytical results from Eqs. (19) and (20) and the full symbols to the numerical simulations. The empty symbols indicate the approximate analytical speed results. The parameter values used are $C_{0}=3.8 \times 10^{7} / \mathrm{ml}, D=8.37 \times 10^{-5} \mathrm{~cm}^{2} / \mathrm{h}, k_{2}=2.47 / \mathrm{h}$, and $Y$ $=2.77 \times 10^{5}$.

is the same procedure we already used in Fig. 1 in Ref. [13] (that figure gives details on the same computation for the case of $\mathrm{T} 7$ viruses infecting $E$. Coli bacteria). This yields $k_{2}=2.47 / \mathrm{h}$ and $Y=2.77 \times 10^{5}$ for VSV infecting BHK cells. The analytical results and numerical simulations for the front speed are shown in Fig. 2(a) using these values. For comparison, in Fig. 2(b) we have used the yield $Y=4389$ proposed in Ref. [17]. All parameters except $Y$ have the same values in Figs. 2(a) and 2(b). Let us now discuss the values of the remaining parameters.

Since the diffusion coefficient $D$ of VSV in agar solutions has not been measured, we performed our analytical and numerical computations using two different values of $D$, namely, that in agar for other viruses $[25,13][D=1.44$ $\times 10^{-4} \mathrm{~cm}^{2} / \mathrm{h}$, full curves in Figs. 2(a) and 2(b)] and the only value of $D$ for VSV available, which refers to a very specific water solution [26] $\left[D=8.37 \times 10^{-5} \mathrm{~cm}^{2} / \mathrm{h}\right.$, dashed curves in Figs. 2(a) and 2(b)].

After VSV viruses infect BHK cells, their progeny leave the cells between $\tau \simeq 2 \mathrm{~h}$ and $\tau \simeq 10 \mathrm{~h}$ after infection (see Fig. 3(a) in [17]). Within this range, the predicted speeds in our Fig. 2(a) are consistent (for $3<\tau<7 \mathrm{~h}$ ) with the observed range, namely, $(6.6-8.0) \times 10^{-3} \mathrm{~cm} / \mathrm{h}[27]$, in spite of the fact that the value of the diffusivity for the VSV virus is rather uncertain because (as mentioned above) it has not been measured through agar [29]. Essentially the same agreement between theory and experiment is also obtained for the value of $Y$ proposed in Ref. [17] [Fig. 2(b)]. In contrast, the nondelayed model $[\tau=0$, as assumed in the model in Ref. [17] corresponding to Eq. (2) in the present paper] yields a very large error, as it is clear from its mismatch with the experimental data [compare the dotted horizontal lines to the shaded rectangle in Figs. 2(a) and 2(b)]. This comparison with the experiments clearly shows the need to take into account the delay-time effect in order to explain the infection speed. This conclusion had not been reached before for VSV infections.

Our approximate solutions for the speed [Eqs. (31) and (32)] make it possible to understand better the differences between Figs. 2(a) and 2(b). If the delay time $\tau$ is large enough, according to Eq. (31) the front speed does not depend on $Y$ neither $\kappa$. Both simulations and exact analytical results agree with the nondependence of the speed on $Y$, since for $\tau>2 \mathrm{~h}$ the curves in Figs. 2(a) and 2(b) are approximately the same. Note also that the approximate Eq. (31) has been derived assuming a high value of $Y$. Because of this, for $\tau<2 \mathrm{~h}$ some differences between the exact and the approximate results arise [specially in Fig. 2(b), because the value of $Y$ is two orders of magnitude smaller than in Fig. 2(a)]. Similarly, the approximate speed for $\tau=0$ [Eq. (32)] is much more accurate for high values of $Y$ [Fig. 2(a)].

In Figs. 1 and 2 we have used the value $k_{1}=1.4$ $\times 10^{-10} \mathrm{~cm}^{3} / \mathrm{h}$ proposed in Ref. [17] for the adsorption rate because the value of $k_{1}$ has not been measured experimentally for VSV infecting BHK cells. However, the former value $k_{1}=1.4 \times 10^{-10} \mathrm{~cm}^{3} / \mathrm{h}$ was obtained by fitting the observations to an extracellular model in Ref. [17]. Because that model does not take into account the role of the delay time $\tau$ [compare Eqs. (2)-(11) above], this value of $k_{1}$ should be regarded as highly uncertain. Therefore, we analyzed the dependence of the front speed on $k_{1}$. We found the very interesting result, shown in Fig. 3, that the value of the front speed is approximately independent of $k_{1}$ over several orders of magnitude (provided that the delay time $\tau$ is taken into account, as first done in the present paper for VSV infections). Indeed, in Fig. 3, the speed remains almost independent of the adsorption rate $k_{1}$ for large enough values of the delay time $\tau$, namely, $\tau>2 \mathrm{~h}$. In contrast, $k_{1}$ becomes a relevant parameter for the case $\tau=0$. This is reasonable intuitively, because a long enough delay $\tau$ in the release of the new generation of viruses from the infected cells will substantially slow down the infection process. Then we should expect the time needed for the parent virus to cross the cell membrane (which is measured by $k_{1}$ ) to become irrelevant. This is indeed observed in Fig. 3 for $\tau>2$ h. The approximate solution given by Eqs. (31) and (32) also supports this conclusion, because for large enough values of $\tau(\beta \gg 1)$ according to Eq. (31) the speed does not depend on $\kappa$, hence neither in $k_{1}$ [whereas for the nondelayed case $(\tau=0)$, Eq. (32) shows a nonlinear dependence on $\kappa$ and therefore on $k_{1}$ ]. This also illustrates that the conclusions obtained from delayed and nondelayed models can be very different. Furthermore, this makes it possible to make quantitative predictions of the front speed in spite of the uncertainty in the value of $k_{1}$, and strongly supports the validity of our Figs. 2(a) and 2(b), because it shows that they would remain almost the same even if the value of $k_{1}$ differed several orders of magnitude from the value used to obtain those figures (namely, $k_{1}=1.4 \times 10^{-10} \mathrm{~cm}^{3} / \mathrm{h}$, from Ref. [17]).

We can also see that there is good agreement between the analytical results from Eqs. (19) and (20) (curves in Figs. 2 and 3 ) and the numerical simulations of Eqs. (15)-(18) (full symbols). 
Relative differences between the speeds in Fig. 3 would not change if a different value of the virus diffusivity $D$ were used. This is due to the fact that in the dimensionless Eq. (19), the parameter $D$ does not appear, thus it is used only when computing the dimensional speed $c$ from the dimensionless one $\bar{c}$ as $c=\bar{c} \sqrt{k_{2} D}$. Thus, using another diffusivity value $\widetilde{D}$ would only change the speeds by the factor $\sqrt{\widetilde{D} / D}$. Hence, we have shown that the only two parameters that have not been measured experimentally (namely, $D$ and $k_{1}$ ) do not affect our conclusion that the role of the delay time cannot be neglected in order to understand VSV infection speeds.

In this section we have shown that the delay time $\tau$ is a strongly significant parameter in models of VSV infection spread. The authors of Ref. [17] fitted a large number of parameters (up to 9). In some cases, this can lead to overfitting the observed phenomena. We also think that it can sometimes lead to unjustified conclusions (e.g., to neglect the role of the delay time, which here we have found to be of utmost importance). In this paper, we have only used two unknown parameters $\left(D\right.$ and $\left.k_{1}\right)$ and shown that they are not strongly relevant to our main conclusions.

\section{CONCLUDING REMARKS}

We have built a simple time-delayed model of VSV infections. In order to do so, we have improved a model previously applied to other kinds of viruses [13]. Our analytical speeds agree well with those from numerical simulations. They are also in agreement with experimentally observed VSV infection speeds [Figs. 2(a) and 2(b)]. Moreover, we have derived approximate front speeds that have lead us to additional understanding on the results and made our conclusions more intuitive.

As expected intuitively, the front speed decreases with increasing values of the delay time $\tau$ [Figs. 2(a) and 2(b)]. We have obtained a strong decrease from $\tau=0$ to $\tau \simeq 1 \mathrm{~h}$, and a smoother decrease for larger values of $\tau$. We have shown that the introduction of the delay time $\tau$ is critically important to account for the front speeds of VSV infections. Indeed, neglecting the delay-time effect (i.e., $\tau=0$ ) leads to an infection front speed one order of magnitude faster than the observed range [Figs. 2(a), 2(b), and 3).

Moreover, we have shown that the infection speed is almost independent of the adsorption rate $k_{1}$ over four orders of magnitude for large enough values of the delay time $\tau$ (Fig. 3), a conclusion not previously reached for any kind of virus infections.

\section{ACKNOWLEDGMENTS}

Funded by the Ministry of Science (Consolider grant No. CSD-2010-00034 and grant No. FIS-2009-13050), the European Commission (Grant No. 28192-FEPRE), and the Generalitat de Catalunya (Grant No. 2009-SGR-374). D.R.A. was supported by the MEC under the FPU program.

\section{APPENDIX A: LOGISTIC GROWTH IN AN HOMOGENEOUS MEDIUM}

As mentioned in Sec. II, Eqs. (2)-(4) do not agree with the experimental data in the so-called one-step growth experiments. To see this, consider the very simple case of a homogeneous medium [so that $\left.\partial^{2}[V](r, t) / \partial r^{2}=0\right]$, composed initially only of infected cells $[[C](t) \simeq 0$ and $[I]$ $\left.(t=0)=[I]_{0}\right]$ and free viruses $\left[[V](t=0)=[V]_{0}\right]$. In such a situation, Eq. (4) becomes simply

$$
\frac{d[I](t)}{d t}=-k_{2}[I](t),
$$

so that

$$
[I](t)=[I]_{0} \exp \left(-k_{2} t\right),
$$

and Eq. (2) simplifies to

$$
\frac{d[V](t)}{d t}=k_{2} Y[I](t)=Y k_{2}[I]_{0} \exp \left(-k_{2} t\right),
$$

thus

$$
[V](t)=[V]_{0}+Y[I]_{0}\left(1-\exp \left[-k_{2} t\right]\right) .
$$

However, this exponential behavior does not agree with the experimental data in the so-called one-step growth experiments, because in those experiments a logistic or S-shape curve is always observed instead (see, e.g., Fig. 1 in Ref. [13]).

As a solution to avoid this inconsistency, it has been proposed [13] to replace Eqs. (2)-(4) by Eqs. (5)-(7). In order to explain this point, let us derive the virus dynamics predicted by the set [Eqs. (5)-(7)] in one-step growth experiments. Taking into account again that those experiments are performed in an homogeneous medium [so that the first term in the right-hand side of Eq. (5) is negligible] and in the absence of uninfected cells [so that $[C](r, t) \simeq 0]$, Eqs. (5)-(7) simplify to

$$
\begin{gathered}
\frac{d[V](t)}{d t}=-Y \frac{d[I](t)}{d t}, \\
\frac{d[I](t)}{d t}=-k_{2}[I](t)\left(1-\frac{[I](t)}{I_{M A X}}\right) .
\end{gathered}
$$

Integration of Eq. (A6) yields

$$
[I](t)=\frac{I_{M A X}}{1+\left(\frac{I_{M A X}}{[I]_{0}}-1\right) \exp \left[k_{2} t\right]},
$$

where $[I]_{0}$ is the value of $[I](t)$ at time $t=0$. Note that this equation leads to $[I](t) \rightarrow 0$ for $t \rightarrow \infty$, as it should (because after a sufficiently long time, all infected cells will have died due to the second of reactions [Eq. (1)]).

On the other hand, integrating Eq. (A5) from $t=0$ to $t$ $\rightarrow \infty$ we come to

$$
[V]_{\infty}=[V]_{0}+Y[I]_{0},
$$

so the increase in the concentration of viruses is limited by the initial concentration of infected cells $[I]_{0}$ and the yield $Y$, 
as it should (because $Y$ viruses per infected cell are produced, according to the second of reactions [Eq. (1)]).

If we integrate Eq. (A5) from $t=0$ to $t$ we obtain, instead of Eq. (A8),

$$
[V](t)=[V]_{0}+Y\left[[I]_{0}-[I](t)\right],
$$

so, again, the increase in virus particles is equal to the decrease in infected cells multiplied by the yield. This is much more reasonable intuitively than Eq. (A4).

Using Eq. (A7) into Eq. (A9) leads us to

$$
[V](t)=[V]_{0}+Y[I]_{0}-\frac{Y I_{M A X}}{1+\left(\frac{I_{M A X}}{[I]_{0}}-1\right) \exp \left[k_{2} t\right]} .
$$

If only a few viruses are present initially $\left([V]_{0} \ll Y[I]_{0}\right.$ or $\left.[V]_{0} \ll[V]_{\infty}\right)$ and the initial concentration of infected cells is close to saturation $\left([I]_{0} \simeq I_{M A X}\right)$, this simplifies to

$$
[V](t)=\frac{Y I_{M A X}}{c_{1} \exp \left[-k_{2} t\right]+1},
$$

where $c_{1} \equiv\left(\frac{I_{M A X}}{[I]_{0}}-1\right)^{-1}$. Equation (A11) is the typical logistic or S-shaped curve which (in contrast to the exponential curve [Eq. (A4)]) agrees very well with experimental data of onegrowth experiments [see Eq. (3) and Fig. 1 in Ref. [13], and Fig. 2 in Ref. [16]]. We conclude that, both intuitively [see the text below Eq. (A9)] and from the perspective of providing good fits to experimental data, Eqs. (5)-(7) are more reasonable than Eqs. (2)-(4).

Notwithstanding the former two strong arguments, the question arises if there is some physical mechanism that can lead to a dynamics with a quadratic saturation term [last term in Eq. (A6)] or, equivalently, to its analytical solution [the logistic expression (A11)]. The answer is that there is at least one such mechanism, namely, to consider that in practice not all viruses spend exactly the same time inside a cell before releasing their progeny (i.e., that the value of the delay time $\tau$ is not the same for all virus particles). Then, if we choose the time origin $t=0$ when the viruses enter the cells, the number of viruses at time $t$ will be

$$
[V](t)=[V]_{0}+(Y-1)[V]_{0} \int_{0}^{t} d \tau \varphi(\tau),
$$

where $\varphi(\tau)$ is the probability distribution of the delay time $\tau$. Note that in the special case that all viruses have exactly the same value of $\tau$, say $\tau_{0}, \varphi(\tau)$ is a Dirac delta centered at $\tau$ $=\tau_{0}$ and Eq. (A12) yields $[V](t)=[V]_{0}$ if $t<\tau_{0}$ and $[V](t)$ $=Y[V]_{0}$ if $t>\tau_{0}$, as it should. If we consider the following delay-time distribution,

$$
\varphi(\tau)=\frac{Y I_{\max }}{(Y-1)[V]_{0}} \frac{c_{1} k_{2} \exp \left[-k_{2} \tau\right]}{\left(c_{1} \exp \left[-k_{2} \tau\right]+1\right)^{2}},
$$

which is bell-shaped (see the full curve in the inset to Fig. 1 in Ref. [15]), using it into Eq. (A12) and integrating yields the logistic Eq. (A11), i.e., the solution to the quadratic expression (A6). This gives a possible physical reason for the logistic solution [Eq. (A11)], and thus for the quadratic evolution Eq. (A6). However, we stress that there are other reasons why such a quadratic evolution equation is more reasonable than a linear one, both intuitively [see the text below Eq. (A9)] and from the perspective of providing better fits to experimental data [see the text below Eq. (A11)]. In any case, the inclusion or not of a quadratic saturation term does not affect the analytical results in this paper, since the infection speeds derived would be the same if the quadratic term [last term in Eqs. (5), (7), and (A6)] were neglected [because after linearization, the last term in Eqs. (15), (17), and (18) do not yield any term in Eq. (19)]. However, let us emphasize an important practical advantage of including this quadratic term, namely, that since Eq. (A11) instead Eq. (A4) gives a much better fit to data, it leads to a more accurate estimate of the value of $k_{2}$ (as we have done in the Comparison to experiment section).

Finally, it is worth to note that $Y$ appears multiplying $k_{2}$ in all reaction-diffusion equations for $[V]$ discussed in this paper [i.e., Eqs. (2), (5), (9), and (11)]. Therefore, the question arises if it is possible to absorb $Y$ into $k_{2}$ and, in this way, get rid of one parameter. The problem is that one-step growth experiments measure $[V](t)$ but not $[I](t)$ (see, e.g., p. 1736 in Ref. [17]). Thus in order to estimate the parameter values, it is necessary to fit Eq. (A11) to the experimental data. But in Eq. (A11) $Y$ no longer multiplies $k_{2}$. For this reason, $Y$ cannot be absorbed into $k_{2}$.

\section{APPENDIX B: TIME-DELAYED REACTION-DIFFUSION EQUATION}

In order to make this paper as much self-contained as possible, in this appendix we give a derivation of the timedelayed reaction-diffusion Eq. (8). Variations in the population number density of viruses are due to two processes: population growth (replication minus adsorption) and dispersal. As usual [21], we Taylor-expand the variation due to population growth,

$$
\begin{aligned}
& |[V](x, y, t+\tau)-[V](x, y, t)|_{g} \\
& \quad=\left.\tau \frac{\partial[V](x, y, t)}{\partial t}\right|_{g}+\left.\frac{\tau^{2}}{2} \frac{\partial^{2}[V](x, y, t)}{\partial t^{2}}\right|_{g}+\cdots \\
& \quad=\tau F(r, t)+\left.\frac{\tau^{2}}{2} \frac{\partial F(r, t)}{\partial t}\right|_{g}+\cdots
\end{aligned}
$$

where $(x, y)$ are Cartesian space coordinates, $\tau$ is the generation time, the subindex $g$ means growth, and we have introduced the growth function as $\left.F(r, t) \equiv \frac{\partial[V]}{\partial t}\right|_{g}$. On the other hand, the variation due to dispersal is [22]

$$
\begin{aligned}
|[V](x, y, t+\tau)-[V](x, y, t)|_{d} \\
=\iint[V]\left(x+\Delta_{x}, y+\Delta_{y}, t\right) \phi\left(\Delta_{x}, \Delta_{y}\right) \mathrm{d} \Delta_{x} \mathrm{~d} \Delta_{y}-[V] \\
\quad \times(x, y, t),
\end{aligned}
$$

where we have introduced the dispersal kernel $\phi\left(\Delta_{x}, \Delta_{y}\right)$, defined as the probability per unit area that a virus particle initially placed at $\left(x+\Delta_{x}, y+\Delta_{y}\right)$ has moved to $(x, y)$ after a 
generation time $\tau$. In a system involving both dispersal and population growth, the total variation is the sum of both contributions,

$$
\begin{aligned}
& {[V](x, y, t+\tau)-[V](x, y, t)} \\
& =\iint[V]\left(x+\Delta_{x}, y+\Delta_{y}, t\right) \phi\left(\Delta_{x}, \Delta_{y}\right) d \Delta_{x} d \Delta_{y}-[V](x, y, t) \\
& \quad+\tau F(r, t)+\left.\frac{\tau^{2}}{2} \frac{\partial F(r, t)}{\partial t}\right|_{g}+\cdots
\end{aligned}
$$

Finally, we Taylor-expand Eq. (B3) up to second order in time and space and assume an isotropic kernel [i.e., $\phi\left(\Delta_{x}, \Delta_{y}\right)=\phi(\Delta)$, with $\left.\Delta=\sqrt{\Delta_{x}^{2}+\Delta_{y}^{2}}\right]$. This yields [18]

$$
\begin{aligned}
\frac{\partial[V]}{\partial t}+\frac{\tau}{2} \frac{\partial^{2}[V]}{\partial t^{2}} & =D\left(\frac{\partial^{2}[V]}{\partial x^{2}}+\frac{\partial^{2}[V]}{\partial y^{2}}\right)+F(r, t)+\left.\frac{\tau}{2} \cdot \frac{\partial F(r, t)}{\partial t}\right|_{g} \\
& \simeq D \frac{\partial^{2}[V](r, t)}{\partial r^{2}}+F(r, t)+\left.\frac{\tau}{2} \frac{\partial F(r, t)}{\partial t}\right|_{g}, \quad(\mathrm{~B} 4)
\end{aligned}
$$

where $D$ is the diffusion coefficient $D=\frac{\left\langle\Delta^{2}\right\rangle}{4 \tau}=\frac{\left\langle\Delta_{x}^{2}\right\rangle}{2 \tau}=\frac{\left\langle\Delta_{y}^{2}\right\rangle}{2 \tau}$. Equation (B4) is the time-delayed Eq. (8) in our model. Note that the last term reads $\left.\frac{\partial F(r, t)}{\partial t}\right|_{g}$ instead of $\frac{\partial F(r, t)}{\partial t}$. This corrects an error in a previous derivation [22] that was later applied to T7 virus infection fronts infecting $E$. Coli bacteria $[13,14]$. That error was due to not including the symbol $\left.\ldots\right|_{g}$ in the last term in Eq. (B1).
[1] N. T. J. Bailey, The Mathematical Theory of Infectious Diseases, 2nd ed. (Griffin, London, 1975).

[2] J. Müller, SIAM J. Appl. Math. 59, 222 (1998).

[3] J. Müller, B. Schönfisch, and M. Kirkilionis, J. Math. Biol. 41, 143 (2000)

[4] J. Müller, M. Kretzschmar, and K. Dietz, Math. Biosci. 164, 39 (2000).

[5] R. Huerta and L. S. Tsimring, Phys. Rev. E 66, 056115 (2002).

[6] L. S. Tsimring and R. Huerta, Physica A 325, 33 (2003).

[7] J. Yin and J. S. McCaskill, Biophys. J. 61, 1540 (1992).

[8] J. Fort, J. Theor. Biol. 214, 515 (2002).

[9] S. P. Brown et al., PLoS Biol. 4, e349 (2006).

[10] S. T. Abedon and R. C. Culler, J. Theor. Biol. 248, 111 (2007).

[11] J. M. Los, P. Golec, G. Wegrzyn, A. Wegrzyn, and M. Los, Appl. Environ. Microbiol. 74, 5113 (2008).

[12] L. Kaliniene, V. Klausa, and L. Truncaite, Arch. Virol. 155, 871 (2010).

[13] J. Fort and V. Méndez, Phys. Rev. Lett. 89, 178101 (2002).

[14] V. Ortega-Cejas, J. Fort, V. Méndez, and D. Campos, Phys. Rev. E 69, 031909 (2004).

[15] J. Fort, J. Pérez, E. Ubeda, and F. J. García, Phys. Rev. E 73, 021907 (2006).

[16] V. Lam, K. A. Duca, and J. Yin, Biotechnol. Bioeng. 90, 793 (2005).

[17] L. Haseltine, V. Lam, J. Yin, and J. B. Rawlings, Bull. Math. Biol. 70, 1730 (2008).

[18] The Laplacian is $\frac{\partial^{2}[V](r, t)}{\partial x^{2}}+\frac{\partial^{2}[V](r, t)}{\partial y^{2}}=\frac{1}{r} \frac{\partial}{\partial r}\left(r \frac{\partial[V](r, t)}{\partial r}\right)=\frac{1}{r} \frac{\partial[V](r, t)}{\partial r}$ $+\frac{\partial^{2}[V](r, t)}{\partial r^{2}}$, but since we are interested in the asymptotic front speed $(r \rightarrow \infty$ and $t \rightarrow \infty)$ this simplifies into $\frac{\partial^{2}[V](r, t)}{\partial r^{2}}$

[19] We try to avoid using unnecessary parameters to explain the experimental front speeds, so we neglect the effects of (i) the reproduction of uninfected cells [i.e., we use $k_{3}=0$ in Eq. (5b) in Ref. [17], as in the extracellular model with no growth in Table 3 in that reference], (ii) the decay of dead cells $\left[k_{4}=0\right.$ in Eq. (5d) in [17]], (iii) age dependencies, etc.

[20] D. Jou, J. Casas-Vázquez, and G. Lebon, Extended Irreversible Thermodynamics (Springer-Verlag, Berlin, 1996).

[21] N. Isern and J. Fort, Phys. Rev. E 80, 057103 (2009).

[22] J. Fort and V. Méndez, Phys. Rev. Lett. 82, 867 (1999).

[23] U. Ebert and W. van Saarloos, Physica D 146, 1 (2000)

[24] L. You and J. Yin, J. Theor. Biol. 200, 365 (1999), Fig. 2.

[25] H. W. Ackermann, Pathol. Biol. (Paris) 24, 359 (1976).

[26] B. R. Ware, T. Raj, W. H. Flygare, J. A. Lesnaw, and M. E. Reichmann, J. Virol. 11, 141 (1973).

[27] The mean of this range was obtained from the slope of a linear fit to Fig. 2B in Ref. [16] (data points labeled N1), and the error of the slope was estimated at the $95 \%$ confidence-level interval by multiplying the standard deviation of the slope by the $t$-distribution $t\left(\frac{0.005}{2}, \nu\right) \simeq 3.2$, with $\nu=5-2=3$ because there are five data points in that figure (see Ref. [28]).

[28] N. R. Draper and H. Smith, Applied Regression Analysis, 2nd ed. (Wiley, New York, 1981), p. 25.

[29] The value of the diffusivity is rather uncertain also because the value of $f \equiv C_{0} / C_{\max }$ necessary to estimate the effective diffusion coefficient $\left(D_{\text {eff }}\right.$ in Ref. [22]) was not measured in Refs. $[16,17]$, so we have had to approximate $D_{e f f} \simeq D$. 